\title{
Cura de orificio de salida de catéter: ciprofloxacino versus suero hipertónico $20 \%$
}

\author{
Maite González Manjón - Virginia Fernández Iñiguez de Heredia - Begoña Capillas Echevarria
}

Unidad de Hemodiálisis. Hospital Santiago Apóstol. Vitoria-Gasteiz

\section{Sra. Directora:}

El aumento del número de pacientes que precisan de hemodiálisis en los últimos años ha hecho que varíe el perfil del paciente renal, incrementándose los pacientes que precisan de la implantación de un catéter permanente para la sesión de hemodiálisis y con ello las complicaciones que de éste se derivan. Una de las complicaciones más frecuentes y que mayor repercusión ocasiona es la infección del orificio de salida. Para la prevención de la infección, la esterilidad en la manipulación del catéter y la cura del orificio de salida del catéter por parte del personal de enfermería son fundamentales. En la unidad de Hemodiálisis del Hospital Santiago Apóstol se realiza la cura del orificio de salida con ciprofloxacino (Septocipro 0tico®), tras limpieza previa con suero fisiológico según el protocolo que se muestra en el anexo 1.

\section{ANEXO 1}

\section{CURA ORIFICIO DE CATÉTER CENTRAL}

Objetivo: prevenir infecciones del catéter central realizando la cura con la máxima asepsia posible.

Material: mascarillas, guantes estériles, gasas, paño estéril, solución salina fisiológica, solución antiséptica Septocipro Otico ß y apósito.

\section{Ejecución:}

1. Informe al paciente sobre la técnica que se va a realizar.

\begin{tabular}{|c|}
\hline Correspondencia: \\
Begoña Capillas Echevarria \\
Servicio de Hemodiálisis \\
Hospital Santiago Apóstol \\
C/ Olaguibel, 29 \\
01004 Vitoria-Gasteiz \\
MARIABEGONA.CAPILLASECHEVARRIA@osakidetza.net \\
\hline
\end{tabular}

2. Coloque al paciente en decúbito supino.

3. Coloque al paciente la mascarilla.

4. Colóquense la mascarilla los dos miembros del equipo que van a realizar la técnica (siempre se realizará la cura del orificio con otro miembro del equipo, auxiliar de enfermería).

5. Colóquese guantes estériles.

6. Proceda a la preparación del campo estéril con la ayuda del otro miembro del equipo.

7. La auxiliar de enfermería que esté ayudando en el procedimiento retira el apósito que cubre el orificio.

8. Limpie la piel con solución salina fisiológica.

9. Seque con gasas estériles.

10. Valoré signos y síntomas de infección, siempre que valore alteraciones en el punto de inserción o en la piel de alrededor debe ponerlo en conocimiento del médico para que tome las medidas encaminadas a evitar problemas más importantes y tome muestra estéril con un bastoncillo para su cultivo.

11. Rocíe con la solución desinfectante (Septocipro) el punto de inserción.

12. Coloque un apósito adecuado a las características de la piel del paciente sobre el punto de inserción.

La bibliografía revisada y las diferentes unidades de hemodiálisis consultadas varían en cuanto a cómo realizar la cura del orificio de salida del catéter, por ello se planteó la necesidad de estudiar la eficacia del ciprofloxacino versus la utilización de suero hipertónico, en la cura del orificio de salida del catéter. Por tanto, nos planteamos estudiar la necesidad o no de la utilización de un antibiótico (ciprofloxacino; Septocipro Otico $®$ ) para la prevención de las infecciones del orificio de salida del catéter permanente.

Se trata de un estudio observacional de cohortes realizado durante cinco meses (abril-agosto del 
2007) en el que la población diana han sido los pacientes portadores de catéter permanente de la unidad de hemodiálisis del Hospital de Santiago Apóstol de Vitoria-Gasteiz. Dicha unidad trataba en el momento del estudio a 56 pacientes de los cuales 15 eran portadores de catéter permanente.

Tras el consentimiento de los pacientes, se les tomaron cultivos nasales, orofaríngeos, así como de orificio de salida de catéter para descartar del estudio a aquellos pacientes con infección previa y a los que eran portadores de algún tipo de patógeno e iniciar su tratamiento correspondiente. El $26,6 \%$ de los pacientes portadores de catéter permanente no dieron su consentimiento para participar en dicho estudio, el $13,3 \%$ fue excluido por presentar infección previa de catéter y el 6,6\% por exitus al inicio del estudio, con lo que la muestra final a estudio fue de 8 pacientes.

Posteriormente se dividieron los pacientes aleatoriamente en 2 grupos. A un grupo se le realizó la cura del orificio de salida con suero hipertónico $20 \%$, mientras que al otro se le realizó la cura del orificio de salida con suero fisiológico más ciprofloxacino. Para ver la evolución y detectar la infección se valoró la aparición de signos de infección tales como dolor, induración, enrojecimiento de la piel, la presencia de costras y la secreción externa.

Del grupo tratado con suero hipertónico uno de ellos mostró infección por Staphylococcus epidermidis (07-06-2007). De entre los que el orificio de salida fue curado con suero fisiológico mas ciprofloxacino, uno mostró varias infecciones; en dos ocasiones por Acinetobacter species (12-04-2007, 29-05-2007), en otra ocasión por Enterococcus faecalis (31-05-2007) y por último por Staphylococcus epidermidis (26-072007), (figura 1). No consideramos los resultados referentes a los cultivos nasales y orofaríngeos debido a que utilizamos mascarilla durante la cura del orificio de catéter.

\section{POBLACION 15 PACIENTES CON CATETER PERMANENTE}

\section{CONSENTIMIENTO}
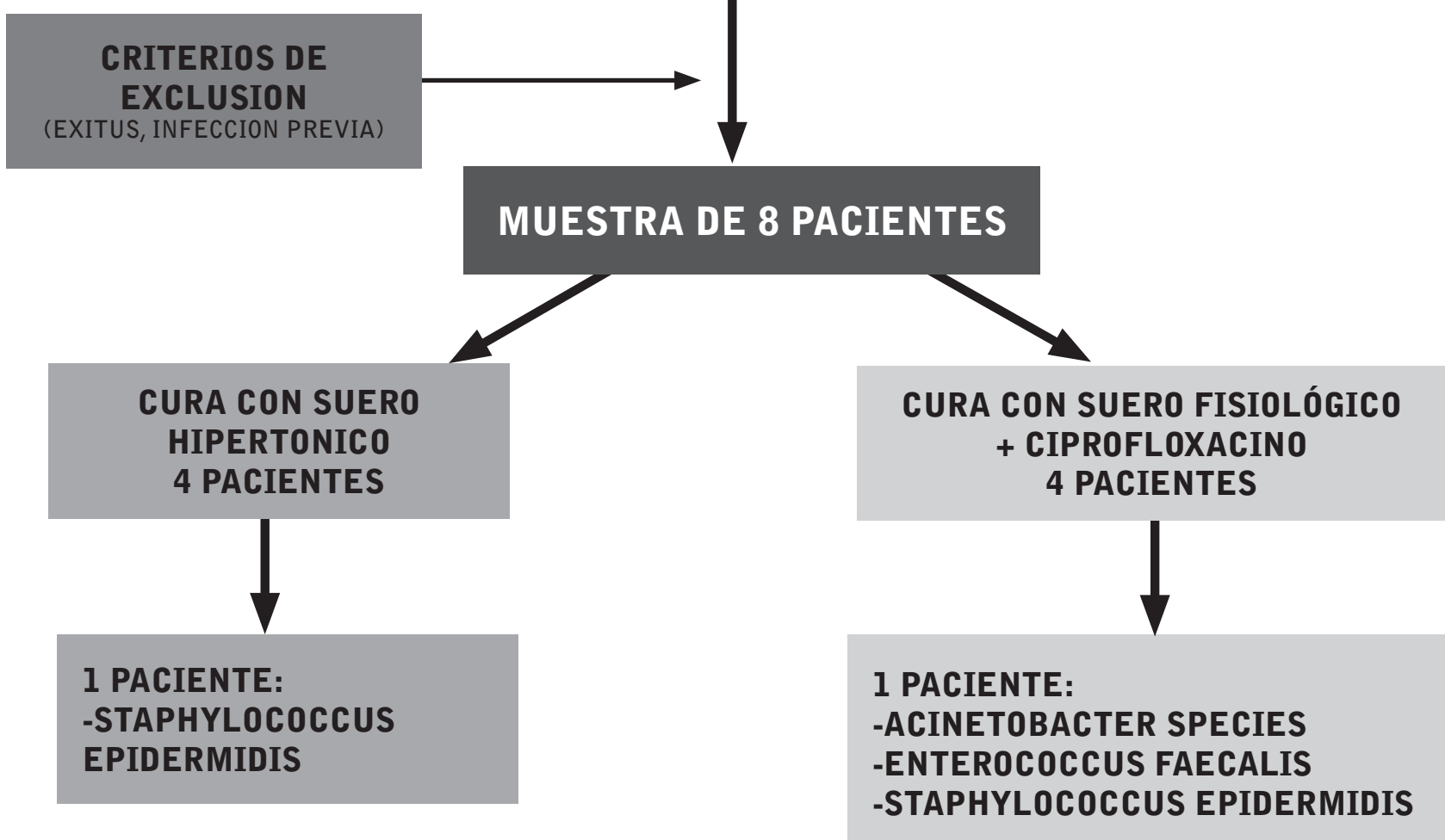

Figura 1. Esquema de actuación y resultados 
La bibliografía consultada respecto al tema del cuidado del orificio de salida de los catéteres en hemodiálisis, muestra que existen discrepancias respecto a la cura de éste. En algunos estudios se hace referencia a la cura con Bactroban ${ }^{\circledR}$, en otros a la cura con suero hipertónico $20 \%$, pero en ninguno a la cura con ciprofloxacino. Por lo que no hay unanimidad en cual es el más indicado. La solución desinfectante utilizada en la cura es importante pero no debemos olvidarnos de la importancia de la manipulación del catéter tanto por parte del personal de enfermería como por parte del propio usuario (higiene...) así como la propia flora de la piel de éste. Además se menciona que la terapia antibiótica no siendo necesaria no estaría indicada por la posibilidad de que se creen resistencias a los antibióticos utilizados en la cura.

En nuestro estudio no se muestran diferencias con respecto al número de infecciones de orificio de salida de catéter entre el grupo curado con suero hipertónico y el grupo curado con suero fisiológico más ciprofloxacino, pero cabe decir que quizá el limitado tamaño de la muestra y el corto periodo del estudio han podido influir. Así mismo hay que añadir que las infecciones del orificio curado con ciprofloxacino mas suero fisiológico pueden estar en relación con varios factores; como el ser portador de VIH y la mala manipulación del catéter por parte del usuario, ya que en varias ocasiones a su llegada a diálisis presentó la funda del catéter mojada.

\section{Bibliografía}

1. Ferre Díaz A. Protocolo de cuidados de catéteres centrales para hemodiálisis. En: Libro de comunicaciones del XXXI Congreso de la Sociedad Española de Enfermería Neurológica; Córdoba 1-4 octubre 2006. Barcelona: HOSPAL; 2006. p.401.

2. Calatrava Fernández $F$, Gómez Montes $M M$, Torres Colomera I. Estudio de las infecciones de catéteres venosos centrales en hemodiálisis. En: Libro de comunicaciones del XXXI Congreso de la Sociedad Española de Enfermería Nefrológica; Córdoba 1-4 octubre 2006. Barcelona: HOSPAL; 2006. p.362.

3. Luna $P$, Castañeda $E$, González V, García-Herrainz $M$, González T, Morell M et al. Estudio comparativo en la cura de catéteres permanentes de hemodiálisis. Rev Soc Esp Enferm Nefrol 2002; Primer trimestre 17: 69-70.

4. Ojeda Fernández E, Gredoria Magias L. Infecciones asociadas a catéteres.Burgos.Disponible en:http:// uninet.edu/cin2000/conferences/ojeda/ojeda.htm

5. Moya Mejia C, Fernández Ros M, Ibeas J, Alcaraz Busqueta J, García García M et al. Monitorización sistemática del catéter permanente: una herramienta útil en el estudio de la tasa de infección y disfunción de dos tipos de catéteres tunelizados para hemodiálisis. Rev Soc Esp Enferm Nefrol. 2006; 9(3):165-171.

6. Manrique Jimeno MP, Sánchez González N, López García MD. Cuidados del orificio de salida de catéteres temporales para hemodiálisis. Comparación de dos métodos. Disponible en:www.seden.org/publicaciones. 